\title{
Tuberculosis at Farmer-Cattle Interface in the Rural Villages of South Gondar Zone of Northwest Ethiopia
}

\author{
Amir Alelign (1D, ${ }^{1,2,3}$ Aboma Zewude, ${ }^{1}$ Beyene Petros, ${ }^{2}$ and Gobena Ameni ${ }^{1}$ \\ ${ }^{1}$ Aklilu Lemma Institute of Pathobiology, Addis Ababa University, P.O. Box 1176, Addis Ababa, Ethiopia \\ ${ }^{2}$ College of Natural Sciences, Department of Microbial, Cellular and Molecular Biology, Addis Ababa University, P.O. Box 1176, \\ Addis Ababa, Ethiopia \\ ${ }^{3}$ College of Natural and Computational Sciences, Department of Biology, Debrebrhan University, P.O. Box 445, Debrebrhan, Ethiopia
}

Correspondence should be addressed to Amir Alelign; aleamiro2009@gmail.com

Received 4 July 2019; Accepted 4 August 2019; Published 16 October 2019

Academic Editor: Alexander S. Apt

Copyright (c) 2019 Amir Alelign et al. This is an open access article distributed under the Creative Commons Attribution License, which permits unrestricted use, distribution, and reproduction in any medium, provided the original work is properly cited.

\begin{abstract}
Background. Tuberculosis (TB) has been an important public health concern in Ethiopia, particularly at areas of human-animal intersection. However, limited epidemiological information is available in this respect in the country. Therefore, the present study was conducted to investigate the transmission of TB at human-cattle interface, associated risk factors and public awareness about the disease at South Gondar Zone, northwest Ethiopia. Methods. A cross sectional study was conducted between March 2015 and April 2018 on 186 farmers and 476 cattle in South Gondar Zone, northwest Ethiopia. Bacteriological examination, region of difference (RD) 9-based polymerase chain reaction (PCR), single intradermal comparative tuberculin test (SICTT), and questionnaire were used for undertaking this study. Results. Culture positivity in farmers was 59.7\% (111/186) and all the culture positive isolates were M. tuberculosis. About $68 \%$ (74/111) of culture positive respondents did not know about the transmission of TB from cattle to human or vice versa. The animal and herd prevalence of bovine TB were 1.5\% (7/476) and 7.4\% (7/95), respectively. Although the result was not statistically significant, the odds of bovine TB in cattle owned by TB positive households was slightly higher than those owned by TB free households (adjusted odds ratio, AOR =1.39; 95\% CI: 0.31-7.10; $p=0.76$ ). Conclusion. Although SIDCTT reactivity was slightly higher in cattle owned by TB positive households, all the human isolates were M. tuberculosis and no M. bovis was isolated from farmers, which could be due to the low prevalence of bovine TB in the area.
\end{abstract}

\section{Introduction}

Tuberculosis (TB) is an infectious disease of humans and animals. The most common cause of human TB is known to be Mycobacterium tuberculosis (M. tuberculosis), and the main cause of TB in animals is Mycobacterium bovis (M. bovis). Zoonotic TB is a form of TB that is caused by M. bovis and transmitted from animals to humans. While reverse zoonotic TB is the form of TB that is caused by M. tuberculosis and is transmitted from humans to animals. M. bovis often causes extra pulmonary TB (EPTB) disease and pulmonary TB (PTB) disease, which is clinically indistinguishable from TB caused by $M$. tuberculosis. In 2016, an estimated 147,000 new cases of zoonotic TB were reported globally, and 12,500 deaths from zoonotic TB. The highest burden of zoonotic TB was reported from the African, followed by the South-East Asian region [1].
The transmission of TB from cattle to human is due to consumption of raw/undercooked infected animal products such as milk and meat or through inhalation due to close contact between the cattle and humans. It is estimated that in countries where pasteurization of milk is rare and bovine TB is common, $10 \%-15 \%$ human cases of $\mathrm{TB}$ are caused by M. bovis [2]. According to some studies from Tanzania, Nigeria, and Uganda, $M$. bovis accounted for $20 \%$ or more of the MTBC isolated from human TB cases [3-5].

Data on TB transmission at the human-animal interface are important in designing a "one health" approach for the control of the disease, particularly, rural settings. In the Amhara Region, north-western Ethiopia, information on the public health risk of zoonotic TB is scarce. Few cross sectional studies conducted in cattle reported animal prevalence of 
$3.55 \%$ and $8.7 \%[6,7]$. In addition, $M$. bovis was isolated from humans in north-western Ethiopia [7,8].

In South Gondar Zone, Amhara Region, north-western Ethiopia, the practices of inhabitants could promote the transmission of TB from cattle to humans or vice versa. However, there is scarcity of epidemiological data on public awareness, risk factors and transmission of TB between humans and their cattle in the Zone. Hence, the present study was conducted to investigate the public awareness, risk factors to bovine TB (BTB) and the potential transmission of TB from cattle to humans or vice versa in South Gondar Zone of north-western Ethiopia.

\section{Materials and Methods}

2.1. Study Area. The study was conducted in South Gondar Zone, north-western Ethiopia. The Zone is located in the Amhara Region, $660 \mathrm{~km}$ north east of Addis Ababa, the Capital of Ethiopia. The Zone is known for its diverse topography ranging from flat and low grazing land to high cold mountains. The altitude of the Zone ranges from 1500 to 3,600 $\mathrm{m}$ above sea level. The average yearly rainfall of the Zone ranged from $700 \mathrm{~mm}$ to $1300 \mathrm{~mm}$ in while the average daily temperature was $17^{\circ} \mathrm{C}$ in 2017 . South Gondar Zone consisted of 10 districts and covers a total area of $14,320 \mathrm{sq} \mathrm{km}$. According to the Central Statistical Agency of Ethiopia, the Zone has a total population of 2,051,738 and a population density of 145.56 [9]. Majority of the population (90.47\%) of the Zone were rural inhabitants. A total of 468,238 households were counted in this Zone, which resulted in an average of 4.38 persons to a household [9].

The majority of the population has depended on subsistence farming and dairy cattle rearing [10]. The Zone has been known for its indigenous milk cattle such as the "Fogera" and "Dera" cattle. Dairying is commonly practiced using small herd size.

2.2. Study Population and Sampling. Human TB cases were recruited randomly from districts health centres, peripheral health posts, zonal and regional government as well as private referal hospitals (Debretabor, Felegehiwot and Gambi hosptals). The human cases included were those active TB patients who visited the health facilities seeking for TBtreatment. The control human subjects were households who did not have TB cases for the last five years and residing in the same villages with the households which have TB cases in their family members. Cattle owned by both TB positive households and TB negative households were tested for bovine TB.

2.3. General Inclusion and Exclusion Criteria. Small scale dairy farmers who were permanently living in the zone for at least two months prior to the study, aged five years and above at the time of the study as well as who were consented and willing to participate in the study were included, whereas, individuals who had no association with cattle, those who were not willing to participate, aged below five years and seriously ill ones were excluded from participating in the study. Both local and cross breed cattle older than six months and owned by households with active TB cases and TB free households were included. Young cattle with age less than six months, clinically sick ones, and cows one month pre-and post partum were excluded from the study.

2.4. Study Design and Sample Size Determination. A comparative cross sectional study was conducted between December 2015 and February 2018. The sample size for this study was calculated with the assumption of a $15 \%$ bovine TB prevalence in $\mathrm{TB}$ positive households and a $5 \%$ bovine $\mathrm{TB}$ prevalence among TB free households, a 95\% confidence interval (CI) with a power of $80 \%$, a ratio of cases to comparison groups of $3: 1$ (based on findings of $15.3 \%$ bovine TB among TB positive households and a 5.9\% BTB among TB free households) [8]. Adding a $10 \%$ nonresponse rate, the required sample size was 105 for TB positive households and 35 TB for free households. However, in the present study, due to several limitations we have only managed to reach out only $63 \mathrm{~TB}$ positive and $32 \mathrm{~TB}$ negative households. Based on this, a total of 476 cattle ( 315 from TB positive households and 161 from TB free households) were tested for bovine TB.

\section{Data Collection}

3.1. Questioner Survey. All human study participants were interviewed with a questionnaire in order to assess their awareness and practices about the zoonotic transmission of TB, and the possible risk factors for the disease. Their socio-demographic characteristics, knowledge, attitudes, and practices towards $\mathrm{TB}$ including their consumption habits of raw milk/meat as well as husbandry practices were asked. Moreover, all herd owners of tuberculin tested cattle were interviewed for possible risk factors of TB positive cattle. Data on age and sex of individual animal, herd size, origin, breed type, recent introduction of new animals into the herd, and keeping of different livestock together were collected. Previous history about BCG vaccination of cattle was recorded.

3.2. Laboratory Methods. After informed consent was obtained, two sputum samples (on-spot and morning) from suspected PTB patients and fine needle aspirate (FNA) samples fromsuspected EPTB cases were collected by trained laboratory technicians and pathologists, respectively.

The samples were first digested and concentrated/ decontaminated by the N-acetyl-L-cysteine-Sodium hydroxide (NALC-NaOH) method. Smears of the final deposits from the various specimens were stained by the Ziehl-Neelsen (ZN) method and examined under oil immersion using a binocular light microscope. All smear positive TB samples were stored at $+4^{\circ} \mathrm{C}$ at the study site and then transported to Regional Health Research Laboratory Center (Bahirdar) and kept at $+4^{\circ} \mathrm{C}$ until bacteriological culture was performed.

Similarly, FNA specimens were collected by pathologists and stored in cryo-tubes in phosphate buffer saline (PBS) with $\mathrm{pH}$ 7.2. ZN staining was performed. AFB-positive specimens were stored at $-20^{\circ} \mathrm{C}$ until mycobacterial culture was performed. 
The samples were processed for culturing according to the standard methods described earlier $[11,12]$. Both sputum and FNA samples were cultured at the Bahir Dar Regional Health Research Laboratory Centre.

To differentiate $M$. tuberculosis from other members of the M. tuberculosis complex (MTBC) species, RD9-based PCR was performed according to protocols previously described [13]. In addition spoligotyping and $M$. tuberculosis drug resistance (MTBDRplus assay) molecular tests were used to differentiate M. tuberculosis from other members of mycobacteria. M. tuberculosis H37Rv, M. bovis bacille Calmette Guérin (BCG) were included as positive controls and water was used as a negative control. Interpretation of the result was based on bands of different sizes (396 base pairs (bp) for M. tuberculosis and $375 \mathrm{bp}$ for M. bovis) as previously described [14].

\section{Animal Study}

4.1. Single Intradermal Comparative Tuberculin Test. Single intradermal comparative tuberculin test (SIDCTT) was performed for detecting TB in cattle. SICTT was done using both bovine and avian purified protein derivatives (PPDs) (Prionics Lelystad B. V., The Netherlands). The tuberculin test measures the hypersensitivity reaction on the skin due to the administered antigens (PPDs).

Two sites were shaved in the middle of the side of the neck, one above the other, separated by about $12 \mathrm{~cm}$ for injection of the two PPDs. The thickness of the skin fold at both injection sites was measured using a caliper and recorded before injection. The sites were injected with $0.1 \mathrm{ml}$ (2000IU) aliquot of bovine PPD and $0.1 \mathrm{ml}$ (2500IU) of avian PPD intradermally. After $72 \mathrm{~h}$, the two sites were measured for change in skin thickness, and the result was interpreted according to international [15] and local [16] criteria.

\section{Data Analysis}

Data were entered in to Excel file format and transferred in to SPSS software version 25 for statistical analysis. Descriptive statistics were used to depict the demographic variables. Chisquare $\left(\chi^{2}\right)$ test was used to test differences in proportions and the association between categorical variables with raw milk consumption habit. Bivariate and multivariate logistic regressions were used to determine the association between background variables with awareness of zoonotic TB and tuberculin reactivity in cattle. Results were considered statistically significant whenever $p$-value was less than $5 \%$.

5.1. Ethics Approval and Consent to Participate. Ethical clearance for the study was obtained from the Ethics Committee of Addis Ababa University, College of Natural and Computational Science (Ref. CNSDO/491/07/15). In addition, written permission was sought from the Amhara Regional Health Bureau Research Ethical Committee (Ref. HRTT/1/271/07). Each study participant consented with a written form and agreed to participate in the study after a clear explanation of the study objectives and patient data confidentiality. In case of participants under the age of 18 years, consent was obtained from their parents/guardians. The animals used in this study were privately owned by the study participants, and a written consent was sought from the owners to take samples from the animals. A high standard veterinary care was taken to minimize cattle suffering during tuberculin test.

\section{Results}

6.1. Tuberculosis in Farmers. Culture positivity was obtained in $59.7 \%(111 / 186)$ of the active TB cases. Of which, 59.5\% (66/111) was isolated from EPTB patients. The molecular typing of culture positive isolates using RD9-based PCR revealed that all isolates had intact RD9 locus and were subsequently classified as M. tuberculosis. No M. bovis was detected (Figure 1). In addition, the spoligotyping and molecular anti-TB drug sensitivity tests also confirmed that all the mycobacterial isolates from human TB patients were M. tuberculosis (data not shown).

6.2. Awarness on Zoonotic Transmission of TB and Food Consumption Habit. About 68\% (74/111) of the respondents did not know about the transmission of TB from cattle to human or vice versa. About 69\% (77/111) of the respondents had the habit of consuming raw milk and other uncooked dairy products (Table 1).

The logistic regression, taking log-odds of awareness about zoonotic transmission as an outcome variable, resulted patient origin, and educational status were observed to be significantly associated $(p<0.05)$ (Table 1$)$. The study participants in Libo kemkem and Simada were 11 and 10 times more aware about the zoonotic tramnsmission of TB as compared to those of Dera District (Libo kemkem vs. Dera AOR $=11.84$; 95\% CI: $1.67-$ 73.2; $p=0.003$ ) and (Simada vs. Dera AOR $=10.47 ; 95 \% \mathrm{CI}$ : $0.72-116.3 ; p=0.038$ ). The odds of having awareness on zoonotic transmission of TB was higher among individuals with secondary school educational level $(\mathrm{AOR}=4.16$; $95 \% \mathrm{CI}$ : $1.05-$ $15.57 ; p=0.029)$ compared to those of illiterates (Table 1 ).

However, other patient characteristics such as age groups, sex, TB history in the family, raw milk consumption habit, and patient category (new or retreatment cases) were not significantly associated with particpants' over all awarness about zoonotic transmission of TB (Table 1).

\section{Tuberculosis in Cattle}

7.1. Characteristics of the Study Cattle. The majority of the cattle were females accounting for $54.2 \%$ (258/476) of the study cattle. Cattle within the age range of 5-10 years had the greatest share $(47 \%)$ from the total cattle tested with a mean age of 5.5 years. Many of the cattle, 447 (94\%), were Zebu breed, while only $29(6 \%)$ of them were cross breed (Table 2$)$. None of the study cattle had previous exposure to BCG vaccination.

7.2. Prevalence of $T B$ in Cattle. Animal prevalence was $1.6 \%(5 / 315)$ and $1.2 \%(2 / 161)$ at $\geq 2 \mathrm{~mm}$ cut-off value in $\mathrm{TB}$ positive and $\mathrm{TB}$ free households, respectively. Using 


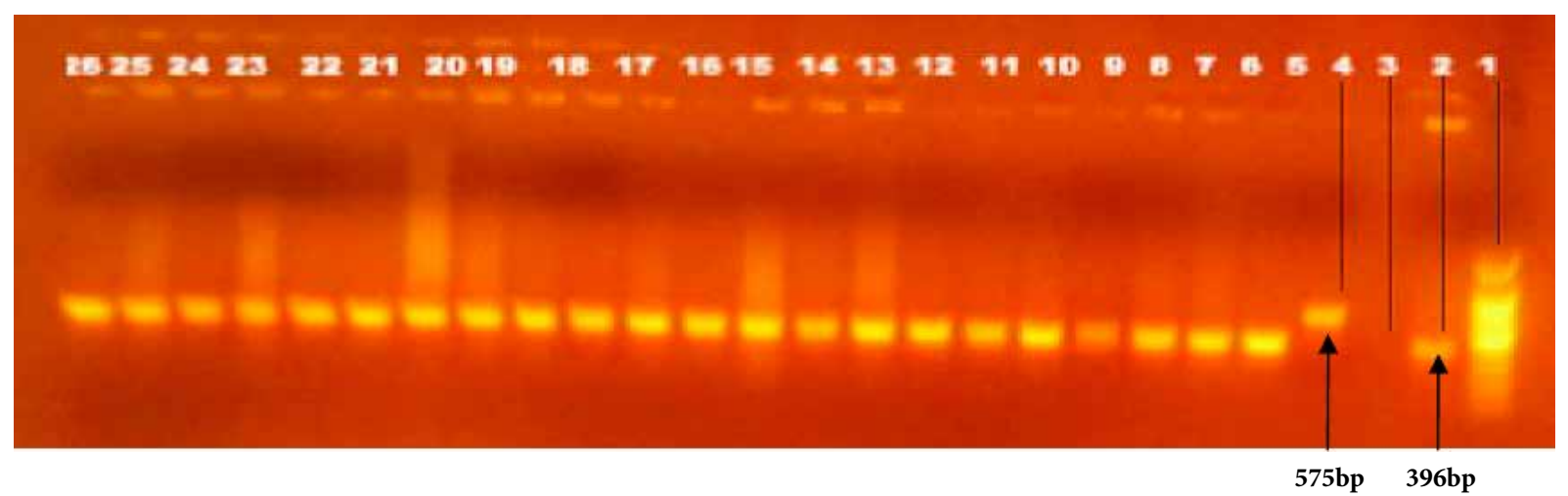

FIGURE 1: Electrophoretic separation of PCR products by RD9 deletion typing. The figure represents only for 22 mycobacteria isolates from sputum and FNA human samples: Lane 1 DNA ladder, 2 M. tuberculosis control (396 base pair), 3 molecular grade water (negative control), $4 M$. bovis control (575 bp), lane 5_26 are culture isolates of $M$. tuberculosis from human tuberculosis patients designated with their sample code as: 5FE1, 6FE2,7FE3,8FE4, 9FE7, 10FE11, 11FE12,12FE15, 13FE16,14FE19,15FE21, 16FE23, 17FE24, 18FE25, 19FE27, 20FE28, 21FE31, 22FE33, 23FE36, 24FE38, 25FE47, 26FE48.

TABLE 1: Association of demographic factors with awarness to zoonotic transmission of TB among AFB culture positive TB patients $(N=111)$, South Gondar Zone, northwest Ethiopia (2015-2017).

\begin{tabular}{|c|c|c|c|c|c|c|}
\hline \multirow{2}{*}{ Demographic factors } & \multirow{2}{*}{$\begin{array}{c}\text { Number of } \\
\text { respondents (\%) }\end{array}$} & \multicolumn{2}{|c|}{ Aware of zoonotic TB } & \multirow{2}{*}{ COR $(95 \% \mathrm{CI})$} & \multirow{2}{*}{$\operatorname{AOR}(95 \% \mathrm{CI})$} & \multirow{2}{*}{$p$-value } \\
\hline & & Yes $(\%)$ & No (\%) & & & \\
\hline \multicolumn{7}{|l|}{ Patient origin } \\
\hline Dera & $26(23.4)$ & $6(23.1)$ & $20(76.9)$ & 1.00 & 1.00 & \\
\hline Ebinat & $10(9)$ & $2(20)$ & $8(80)$ & $0.83(0.13-5.03)$ & $0.66(0.39-1.28)$ & 0.842 \\
\hline Este & $15(13.5)$ & $1(6.7)$ & $14(93.3)$ & $0.23(0.02-2.20)$ & $0.25(0.01-2.53)$ & 0.178 \\
\hline Farta & $9(8.1)$ & $2(22.2)$ & $7(77.8)$ & $0.95(0.15-5.86)$ & $0.63(0.02-5.53)$ & 1.00 \\
\hline Fogera & $35(31.5)$ & $13(37.1)$ & $22(62.9)$ & $1.96(0.62-6.16)$ & $1.99(0.58-8.02)$ & 0.240 \\
\hline Gayint & $6(5.4)$ & $3(50)$ & $3(50)$ & $3.33(0.52-21.03)$ & $3.24(0.31-21.0)$ & 0.186 \\
\hline Libo kemkem & $7(6.3)$ & $5(71.4)$ & $2(28.6)$ & $11.66(1.89-71.79)$ & $11.84(1.67-73.2)$ & 0.003 \\
\hline Simada & $3(2.7)$ & $2(66.7)$ & $1(39.3)$ & $10.0(0.87-114.74)$ & $10.47(0.72-116.3)$ & 0.038 \\
\hline \multicolumn{7}{|l|}{ Age group (year) } \\
\hline$<18$ & $10(9)$ & $5(50)$ & $5(50)$ & 1.00 & 1.00 & \\
\hline $18-30$ & $42(37.8)$ & $17(40.5)$ & $25(59.5)$ & $0.68(0.17-2.71)$ & $0.64(0.13-2.94)$ & 0.583 \\
\hline $31-43$ & $35(31.5)$ & $8(22.9)$ & $27(77.1)$ & $0.29(0.06-1.28)$ & $0.32(0.04-1.42)$ & 0.094 \\
\hline $44-56$ & $18(16.2)$ & $5(27.8)$ & $13(72.2)$ & $0.38(0.07-1.92)$ & $0.46(0.09-2.31)$ & 0.239 \\
\hline$>56$ & $6(5.4)$ & $2(33.3)$ & $4(66.7)$ & $0.50(0.06-4.09)$ & $0.51(0.07-5.21)$ & 0.515 \\
\hline \multicolumn{7}{|l|}{ Sex } \\
\hline Male & $58(52.3)$ & $18(31.0)$ & $40(69.0)$ & 1.00 & 1.00 & \\
\hline Female & $53(47.7)$ & $19(35.8)$ & $34(64.2)$ & $1.24(0.56-2.73)$ & $1.16(0.49-2.97)$ & 0.590 \\
\hline \multicolumn{7}{|l|}{ Education status } \\
\hline Illiterate & $73(65.8)$ & $22(30.1)$ & $51(69.9)$ & 1.00 & 1.00 & \\
\hline Adult education & $5(4.5)$ & $1(0.2)$ & $4(99.8)$ & $0.57(0.06-5.48)$ & $0.23(0.04-5.53)$ & 0.630 \\
\hline Primary level & $18(16.2)$ & $4(22.2)$ & $14(77.8)$ & $0.66(0.19-2.24)$ & $0.62(0.08-2.62)$ & 0.505 \\
\hline Secondary level & $11(9.9)$ & $7(63.6)$ & $4(36.4)$ & $4.05(1.07-15.28)$ & $4.16(1.05-15.57)$ & 0.029 \\
\hline Higher level & $4(3.6)$ & $3(0.75)$ & $1(0.25)$ & $6.95(0.68-70.60)$ & $6.52(0.62-70.71)$ & 0.062 \\
\hline \multicolumn{7}{|l|}{ Raw milk consumption } \\
\hline Yes & $77(69.4)$ & $27(35.1)$ & $50(64.9)$ & 1.00 & 1.00 & \\
\hline No & $34(30.6)$ & $10(29.4)$ & $24(70.6)$ & $0.71(0.29-1.69)$ & $0.71(0.26-1.88)$ & 0.441 \\
\hline \multicolumn{7}{|l|}{ Patient category } \\
\hline New & $91(82.0)$ & $29(31.9)$ & $62(68.1)$ & 1.00 & 1.00 & \\
\hline${ }^{*}$ Retreatment & $20(18.0)$ & $8(40.0)$ & $12(60.0)$ & $1.42(0.52-3.86)$ & $1.38(0.42-3.93)$ & 0.484 \\
\hline
\end{tabular}

${ }^{*}$ Retreatment: A patient who remained smear positive or became again smear positive at the end of five months or later after commencing treatment. COR: Crude odds ratio, AOR: Adjusted odds ratio, CI: Confidence interval. 
TABLE 2: Association of host risk factors with bovine tuberculin test reactivity in cattle based on a $\geq 2 \mathrm{~mm}$ cut-off value, South Gondar Zone, northwest Ethiopia (2015-2018).

\begin{tabular}{|c|c|c|c|c|c|c|}
\hline \multirow{2}{*}{ Characteristics } & \multicolumn{2}{|c|}{ Tuberculin test $(N=476)$} & \multirow{2}{*}{ Total (\%) } & \multirow{2}{*}{ COR $(95 \% \mathrm{CI})$} & \multirow{2}{*}{ AOR (95\% CI) } & \multirow{2}{*}{$p$-value } \\
\hline & Positive & Negative & & & & \\
\hline \multicolumn{7}{|c|}{ Age of cattle (years) } \\
\hline$<5$ & 1 & 160 & $161(33.8)$ & 1 & & \\
\hline $5-10$ & 5 & 203 & $208(43.7)$ & $4.11(0.47-35.55)$ & $3.10(0.35-35.69)$ & 0.16 \\
\hline$>10$ & 1 & 106 & $107(22.5)$ & $1.58(0.09-25.45)$ & $1.69(0.16-25.93)$ & 0.74 \\
\hline \multicolumn{7}{|l|}{ Sex } \\
\hline Male & 2 & 215 & $217(45.6)$ & 1 & & \\
\hline Female & 5 & 254 & $259(54.4)$ & $2.11(0.40-11.01)$ & $2.16(0.35-12.21)$ & 0.36 \\
\hline \multicolumn{7}{|l|}{ Breed type } \\
\hline Local & 6 & 441 & $447(94.0)$ & 1 & & \\
\hline Cross & 1 & 28 & $29(6.0)$ & $2.62(0.30-22.56)$ & $2.67(0.25-23.74)$ & 0.36 \\
\hline \multicolumn{7}{|l|}{ Source } \\
\hline Homebred & 5 & 364 & $369(77.3)$ & 1 & & \\
\hline Purchased & 2 & 105 & $107(22.7)$ & $1.38(0.27-7.25)$ & $1.38(0.17-7.55)$ & 0.69 \\
\hline \multicolumn{7}{|l|}{ Body condition } \\
\hline Poor & 1 & 196 & $197(41.4)$ & 1 & & \\
\hline Medium & 3 & 204 & $207(43.5)$ & $2.88(0.29-27.94)$ & $3.00(0.27-28.38)$ & 0.33 \\
\hline Good & 3 & 69 & $72(15.1)$ & $8.52(0.87-83.29)$ & $8.53(0.85-83.34)$ & 0.02 \\
\hline \multicolumn{7}{|c|}{ Household TB status } \\
\hline Negative & 2 & 159 & $161(33.8)$ & 1 & & \\
\hline Positive & 5 & 310 & $315(66.2)$ & $1.28(0.24-6.68)$ & $1.39(0.31-7.10)$ & 0.76 \\
\hline
\end{tabular}

N: number of total cattle tested. COR: crude odds ratio, AOR: Adjusted odds ratio, CI: confidence interval.

the same cut-off value, $7.9 \%(5 / 63)$ and $6.3 \%(2 / 32)$ herd prevalence was recorded in cattle owned by TB positive and TB free households, respectively. The overall animal and herd prevalence was $1.5 \%(7 / 476)$ and $7.4 \%(7 / 95)$, respectively. However, none of the tested cattle were positive for bovine $\mathrm{TB}$ at the international cut-off value of $>4 \mathrm{~mm}$.

7.3. Risk Factors for Bovine TB. Risk factor analysis to the occurrence of bovine TB in cattle revealed that age groups between 5 and 10 years were more reactive, but not statistically significant $(P>0.05)$, to tuberculin test than younger age groups $(\mathrm{AOR}=3.1 ; 95 \% \mathrm{CI}: 0.35-35.69 ; p=0.16)$. Cattle with apparently $\operatorname{good}(\mathrm{AOR}=8.53 ; 95 \% \mathrm{CI}: 0.85-83.34 ; p=0.02)$ and medium (AOR $=3.00$; 95\% CI: $0.27-28.38 ; p=0.33)$ body conditions were more likely to be reactive to the tuberculin test as compared to those with apparently poor body condition, and the difference was statistically significant $(p<0.05)$ (Table 2$)$. Although the difference was not statistically significant $(p>0.05)$, the odds of bovine TB that cattle owned by TB positive cases were slightly higher than those owned by TB free households $(\mathrm{AOR}=1.39 ; 95 \% \mathrm{CI}: 0.31-7.10 ; p=0.76)$. Despite the observed differences, sex, breed type, source of cattle, and households TB status were not significantly associated $(p>0.05)$ with the occurrence of BTB in the present study (Table 2).

7.4. Zoonotic Transmission of TB. In the present study, molecular typing of culture positive isolates using RD9-based PCR confirmed that all the human isolates were $M$. tuberculosis (Figure 1). Furthermore, no M. bovis was detected even from those TB patients who owned tuberculin reactor cattle. Hence, this study did not reveal evidence of direct transmission of tuberculosis from cattle to their closely associated owners.

\section{Discussion}

The identification of M. tuberculosis as the only Mycobacterium species in the present study, using RD9-based PCR, was in agreement with previous reports in other parts of Ethiopia in which all or the majority of the isolates found from human $\mathrm{TB}$ cases were $M$. tuberculosis [6, 17-19], suggesting a minimal contribution of $M$. bovis to the overall burden of human TB. In contrast, previous studies conducted in large scale commercial farms and pastoral communities suggested the contribution of $M$. bovis to the overall burden of TB in humans [20, 21]. The reason for the difference in Mycobacterium species prevalence in this study and previous studies might be due to the low $\mathrm{TB}$ infection rate in cattle owned by smallholder farmers that participated in the present study.

Although zoonotic transmission of $M$. bovis from cattle to famers was expected, all the human isolates were M. tuberculosis. Nonetheless, previous study conducted in and around Bahir Dar City [8], Borena Zone [20] and Afar Region reported the isolation of M. bovis from human TB cases. It has been well established in the literature that the prevalence of human TB caused by $M$. bovis in specific geographic region is directly proportional to the prevalence of bovine TB in that specific geographic region [22]. In the present study, the prevalence of bovine TB was very low and hence the chance of its transmission to humans is minimal. 
Nevertheless, the awareness of farmers about zoonotic transmission of TB was low and thus was similar to the magnitude of awareness recorded by previous studies conducted in Ethiopia $[8,21,23]$ and in other countries including Zambia and Zimbabwe $[24,25]$. The poor awarness of farmers on the transmission of zoonotic TB to them could pose risk of infection by zoonotic pathogens including $M$. bovis, suggesting a need for an awareness creation campaign about zoonotic TB in the study area. On the other hand, in contrast to the low awareness of the farmers included in the present study, farmers in Cameroon and Malawi had good awareness on the zoonotic TB and its transmission $[26,27]$.

Although $M$. bovis was not isolated from the farmers with active TB, majority of culture posistive TB patients had the habit of consuming raw milk. This observation is similar to the observation of previous studies conducted in different parts of Ethiopia [8,28-30]. The higher preference of raw milk consumption in Ethiopia could be associated to culture, its taste, availability in the local market, an easy access from a door to door supply by farmers and lower price [31].

The animal and herd prevalence of bovine TB at a severe cut-off value of SICTT were low in South Gondar Zone of north-western Ethiopia. In agreement with the prevalence report of this study, low prevalence of bovine TB was reported in and around Bahir Dar City and Yeki District of southern Ethiopia $[6,32]$. On the other hand, higher prevalence of bovine TB was reported in and around other cities of Ethiopia $[16,33-36]$. These variations in the prevalence of bovine TB are associated with the breed of cattle kept and the type of husbandry under which the cattle are kept. Previous studies in Ethiopia have indicated that Bos taurus breed is more susceptible to bovine TB as compared to Bos indicus breed [37]. In the present study about $94 \%$ of the study cattle were local Zebu breeds, which might be the reason for the low power detection of tuberculin reactivity even at a cut-off value of $2 \mathrm{~mm}$. Moreover, it was observed that cattle kept in intensive farms are more susceptible to bovine TB as compared to cattle kept in extensive farms [37]. In addition, it was well established that the prevalence of bovine TB is directly associated with the herd size [37]. Thus, the observation of low prevalence bovine TB in the present study is not surprising as all the study cattle were Bos indicus and were also kept in extensive farming; both of which do not favour the occurrence and transmission of bovine TB. Furthermore, all the herds included in the present study were small (ranging from 3 to 10 cattle per herd) and thus did not favour the transmission of bovine TB.

Nevertheless, although the overall prevalence of bovine TB recorded by the present study was low, it was relatively higher in cattle owned by TB positive households than cattle owned by TB free households. But the difference of prevalence between the two groups was not statistically significant. Other earlier studies reported higher prevalence of bovine $\mathrm{TB}$ in cattle owned by households with active TB cases than TB free households [7, 34, 38, 39]. Such observation could suggest the existence of TB transmission between cattle and their owners. The transmission could be zoonotic (transmission of $M$. bovis from cattle to humans) or reverse zoonotic (transmission of M. tuberculosis from humans to cattle). In the present study, isolation of mycobacteria was not done from cattle and diagnosis of bovine TB was made by SICTT. On the other hand, all the human isolates were $M$. tuberculosis. This could imply that $M$. tuberculosis might have been transmitted to cattle from their owners and positivity to SICTT was due to sensitization to infection with $M$. tuberculosis as it was observed earlier by other authors $[13,38,40]$.

The present study has some limitations in conducting pathological examination and there by strain identification of mycobacteria isolates from tuberculin reactor cattle, which could give more power to infer TB transmission between humans and cattle.

\section{Conclusion}

All the human isolates recovered from farmers with active TB cases were $M$. tuberculosis and no $M$. bovis was isolated. Moreover, the overall prevalence of bovine TB in the area was low; but it was slightly higher in cattle owned by households with active TB cases than in cattle owned with active TB free households; which could suggest the presence of zoonotic and or reverse zoonotic transmission of TB between cattle and their owners. This could also be exacerbated by the low level of awareness of the farmers on the transmission of mycobacterial species between cattle and their owners.

\section{Data Availability}

All the datasets on which our conclusions relayed on were presented in the main section of this manuscript.

\section{Conflicts of Interest}

The authors declare that they have no conflicts of interest.

\section{Funding}

The financial support for the study was obtained from Addis Ababa University (Grant Ref. No. 0162230106072100101). The study was also partially supported by the National Institute of Health (NIH, USA) through its H3Africa Consortium Program (Grant Ref. No. U01HG007472-01).

\section{Acknowledgments}

We would like to thank all laboratory working staff and administrators at the Regional Health research Center, Bahirdar and ALIPB, Addis Ababa University, for their support in the development of this study. We also greatly thank health officials, laboratory workers, and study participants in the study area, without whom this study would have not been completed.

\section{Supplementary Materials}

Appendix Informed Consent form for individuals who attend health centers/hospital in South Gondar Zone of 
the Amhara Region, and who are inviting to participate in research on tuberculosis. The title of the research project is "Tuberculosis in Farmers and Their Cattle in Smallholder Farming System in South Gondar Zone of northwest Ethiopia: Epidemiology and Drug Sensitivity Profiles." (Supplementary Materials)

\section{References}

[1] World Health Organization, "Global zoonotic tuberculosis report 2017," Geneva, Switzerland, 2017, http://www.who.int/ tb/areas-of-work/zoonotic-tb/en.

[2] D. A. Ashford, E. Whitney, P. Raghunathan, and O. Cosivi, "Epidemiology of selected mycobacteria that infect humans and other animals," Revue Scientifique et Technique de l'OIE, vol. 20, no. 1, pp. 325-337, 2001.

[3] S. Cleaveland, D. J. Shaw, G. Mfinanga et al., "Mycobacterium bovis in rural Tanzania: risk factors for infection in human and cattle populations," Tuberculosis, vol. 87, no. 1, pp. 30-43, 2007.

[4] J. D. Mawak, N. E. Gomwalk, C. S. S. Bello, and Y. T. KandakaiOlukemi, "Human pulmonary infections with bovine and environment (atypical) mycobacteria in Jos Nigeria," Ghana Medical Journal, vol. 40, no. 4, pp. 132-136, 2006.

[5] J. Oloya, J. Opuda Asibo, R. Kazwala et al., "Mycobacteria causing human cervical lymphadenitis in pastoral communities in the Karamoja region of Uganda," Epidemiology and Infection, vol. 136, no. 5, pp. 636-643, 2008.

[6] A. Nuru, G. Mamo, L. Teshome et al., "Bovine tuberculosis and its risk factors among dairy cattle herds in and around Bahir Dar City, Northwest Ethiopia," Ethiopian Veterinary Journal, vol. 19, no. 2, pp. 27-40, 2015.

[7] A. Mengistu, F. Enqusselasie, A. Aseffa, and D. Beyene, "Bovine tuberculosis in rural Ethiopia: a comparative cross-sectional study on cattle owned by households with and without tuberculosis," Mycobacterial Diseases, vol. 5, no. 4, 2015.

[8] A. Nuru, G. Mamo, A. Zewude et al., "Preliminary investigation of the transmission of tuberculosis between farmers and their cattle in smallholder farms in northwestern Ethiopia: a crosssectional study," BMC Research Notes, vol. 10, no. 1, Article ID 31, 2017.

[9] Central Statistics Agency (Ethiopia), "Summary and statistical report of the population and housing census: population size by age and sex," Addis Ababa, Ethiopia, 2007, https://www. scribd.com/doc/28289334/Summary-and-Statistical-Reportof-the-2007.

[10] Ethiopia Demographic and Health Survey, "Central Statistical Agency 2011 Ethiopia and ICF International Calverton, Maryland, USA Addis Ababa," https://www.unicef.org/ethiopia/ ET_2011_EDHS.pdf.

[11] Federal Ministry of Health (Ethiopia), "Tuberculosis, Leprosy and TB/HIV Prevention and Control Programme. Addis Ababa, Ethiopia," 4th edition, 2008, http://www.who.int/hiv/pub/ guidelines/ethiopia_tb.pdf.

[12] World Health Organization, "Laboratory services in tuberculosis control: culture. Part III, Geneva, Switzerland 1998," https://apps.who.int/iris/bitstream/10665/65942/3/ WHO_TB_98.258_\%28part3\%29.pdf.

[13] S. Berg, R. Firdessa, M. Habtamu et al., "The burden of mycobacterial disease in Ethiopian cattle: implications for public health," PLoS One, vol. 4, no. 4, e5068 pages, 2009.
[14] L. M. Parsons, R. Brosch, S. T. Cole et al., "Rapid and simple approach for identification of Mycobacterum tuberculosis complex isolates by PCR-based genomic deletion analysis," Journal of Clinical Microbiology, vol. 40, no. 7, pp. 2339-2345, 2002.

[15] Office International des, "Epizooties Terrestrial manual: World Health Organization for Animal Health, Paris. 2009," https://www.devex.com/organizations/world-organisation-foranimal-health-oie-48619.

[16] G. Ameni, G. Hewinson, A. Aseffa, D. Young, and M. Vordermeier, "Appraisal of interpretation criteria for the comparative intradermal tuberculin test for the diagnosis of bovine tuberculosis in Central Ethiopia," Clinical and Vaccine Immunology, vol. 15, no. 8, pp. 1272-1276, 2008.

[17] B. Diriba, T. Berkessa, G. Mamo, Y. Tedla, and G. Ameni, "Spoligotyping ofmultid-rug-resistant Mycobacteriumtuberculosis isolates in Ethiopia," International. Journal of Tuberculosis and Lung Disease, vol. 17, no. 2, pp. 246-250, 2013.

[18] M. Belay, G. Ameni, G. Bjune, D. Couvin, N. Rastogi, and F. Abebe, "Strain diversity of Mycobacterium tuberculosis isolates from pulmonary tuberculosis patients in Afar pastoral region of Ethiopia," BioMedical Research International, vol. 2014, Article ID 238532, pp. 1-12, 2014.

[19] T. Debebe, A. Admassu, G. Mamo, and G. Ameni, "Molecular characterization of Mycobacterium tuberculosis isolated from pulmonary tuberculosis patients in Felege Hiwot Referral Hospital, northwest Ethiopia," Journal of Microbiology Immunology and Infection, vol. 47, no. 4, pp. 333-338, 2014.

[20] B. Gumi, E. Schelling, S. Berg et al., "Zoonotic transmission of tuberculosis between pastoralists and their livestock in SouthEast Ethiopia," Eco Health, vol. 9, no. 2, pp. 139-149, 2012.

[21] M. Tibebu, W. Mekonnen, T. Awoke et al., "A high prevalence of tuberculosis among dairy farm workers in Addis Ababa and its surroundings," Mycobacterial Diseases, vol. 4, no. 1, p. 139, 2014.

[22] O. Cosivi, M. Grange, J. Daborn, C. Raviglione, T. Fujikura, and D. Cousins, "Zoonotic tuberculos-is due to Mycobacterium bovis in developing countries," Emerging Infectious Diseases, vol. 4, no. 1, pp. 59-70, 1998.

[23] B. Temesgen, M. Shigut, T. Hailemariam, and E. Chali, "Assessment of community awareness towards zoonotic tuberculosis in West Shoa, Ethiopia," Clinical Medicine Research, vol. 6, no. 2, pp. 37-42, 2017.

[24] M. Munyeme, B. Muma, H. M. Munang'Andu, C. Kankya, E. Skjerve, and M. Tryland, "Cattle owners' awareness of bovine tuberculosis in high and low prevalence settings of the wildlifelivestock interface areas in Zambia," BMC Veterinary Research, vol. 6, no. 1, p. 21, 2010.

[25] D. Mosalagae, D. M. Pfukenyi, and G. Matope, "Milk producers' awareness of milk borne zoonoses in selected smallholder and commercial dairy farms of Zimbabwe," Tropical Animal Health and Production, vol. 43, no. 3, pp. 733-739, 2011.

[26] J. Ndukum, C. Kudi, G. Bradley, N. Ane-Anyangwe, S. Fon-Tebug, and J. Tchoumboue, "Prevalence of bovine tuberculosis in abattoirs of the littoral and Western highland regions of Cameroon: a cause for public health concern," Veterinary Medicine International, vol. 2010, Article ID 495015, pp. 1-8, 2010.

[27] S. F. Tebug, G. R. Njunga, M. G. Chagunda, J. P. Mapemba, J. Awah-Ndukum, and S. Risk Wiedemann, "knowledge and preventive measures of smallholder dairy farmers in northern Malawi with regard to zoonotic brucellosis and bovine tuberculosis," Onderstepoort Journal of Veterinary Research, vol. 81, no. 1, 2014. 
[28] W. Tigre, G. Alemayehu, T. Abetu, and B. Deressa Gelalcha, "Preliminary study on public health implication of bovine tuberculosis in Jimma town South Western Ethiopia," Global Veterinaria, vol. 6, no. 4, pp. 369-373, 2011.

[29] H. K. Abraham, S. Dessalegn, M. Aklilu, and M. Pal, "Knowledge, attitude and practice towards human and bovine tuberculosis among high school students in Addis Ababa, Ethiopia," International Journal of Livestock Research, vol. 5, no. 1, pp. 1-11, 2015.

[30] L. G. Gebremichael, B. S. Fanta, S. M. Abay, and C. D. Subas, "Anti-tuberculosis drug resistance in Ethiopia: a mata- analysis," Journal of Drug Delivery and Therapeutics, vol. 4, no. 3, pp. 154-163, 2014.

[31] The Netherlands Development Organization, "Dairy investment opportunities in EthiopiaAddis Ababa: 2008," 148 pages, http://www.primeethiopia.org/wpcontent/uploads/2015/03/ dairy_investment_opportunities_in_ethiopia.pdf.

[32] P. Admasu, W. Berihun, and A. Niguse, "Prevalence of bovine tuberculosis in dairy cattle of Yeki district, Southern Ethiopia," African Journal of Basic and Applied Sciences, vol. 6, no. 5, pp. 135-140, 2014.

[33] G. Ameni and E. Aklilu, "Bovine tuberculosis on small-scale dairy farms in Adama Town, central Ethiopia, and farmer awareness of the disease," Revue Scientifique et Technique-Office International des Epizooties, vol. 26, no. 3, pp. 711-719, 2007.

[34] A. Regassa, G. Medhin, and G. Ameni, "Bovine tuberculosis is more prevalent in cattle owned by farmers with active tuberculosis in central Ethiopia," The Veterinary Journal, vol. 178, no. 1, pp. 119-125, 2008.

[35] W. Tsegaye, A. Aseffa, A. Mache, Y. Mengistu, B. Stefan, and G. Ameni, "Conventional and molecular epidemiology of bovine tuberculosis in dairy farms in Addis Ababa city, the capital of Ethiopia," The International Journal of Applied Research in Veterinary Medicine, vol. 8, no. 2, pp. 143-151, 2010.

[36] R. Firdessa, R. Tschopp, A. Wubete et al., "High prevalence of bovine tuberculosis in dairy cattle in central Ethiopia: implications for the dairy industry and public health," PLoS One, vol. 7, no. 12, p. e52851, 2012.

[37] G. Ameni, A. Aseffa, H. Engers, D. Young, G. Hewinson, and M. Vordermeier, "Cattle husbandry in Ethiopia is a predominant factor affecting the pathology of bovine tuberculosis and gamma interferon responses to Mycobacterial antigens," Clinical and Vaccine Immunology, vol. 13, no. 9, pp. 1030-1036, 2006.

[38] G. Ameni, K. Tadesse, E. Hailu et al., "Transmission of Mycobacterium. tuberculosis between farmers and cattle in central Ethiopia," PLoS One, vol. 8, no. 10, e76891 pages, 2013.

[39] F. Tamiru, M. Hailemariam, and W. Terfa, "Preliminary study on prevalence of bovine tuberculo-sis in cattle owned by tuberculosis positive and negative farmers and assessment of zoonotic awareness in Ambo and Toke Kutaye districts, Ethiopia," Journal of Veterinary Medicine and Animal Health, vol. 5, pp. 288-295, 2013.

[40] G. Ameni, M. Vordermeierb, R. Firdessa et al., "Mycobacterium tuberculosis infection in grazing cattle in central Ethiopia," The Veterinary Journal, vol. 188, no. 3, pp. 359-361, 2011. 


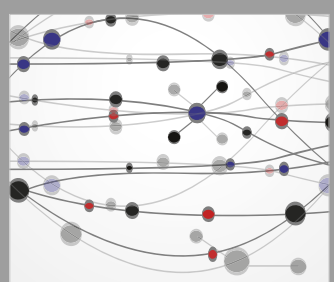

The Scientific World Journal
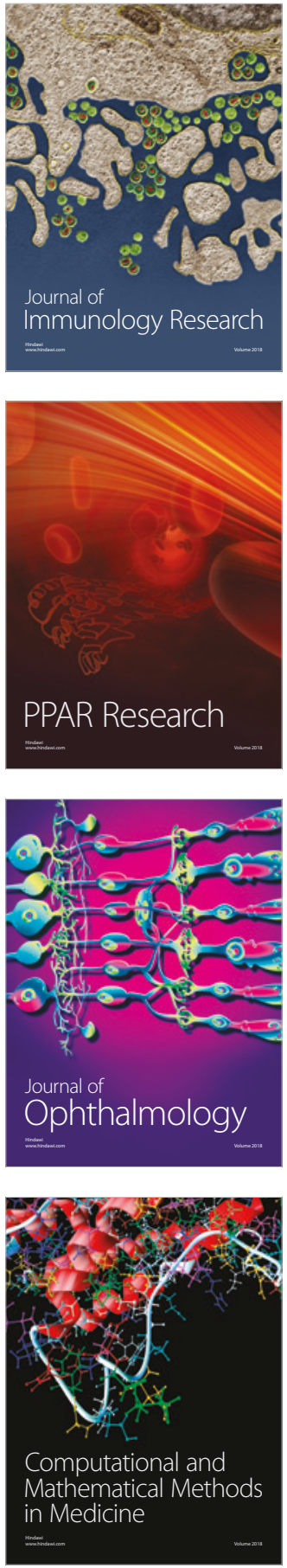

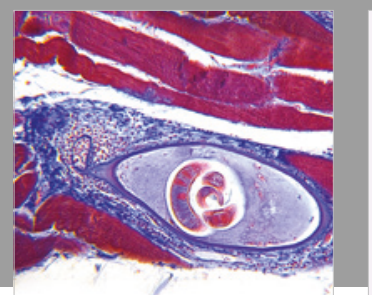

Gastroenterology Research and Practice

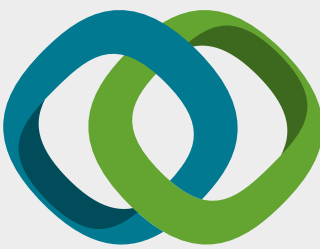

\section{Hindawi}

Submit your manuscripts at

www.hindawi.com
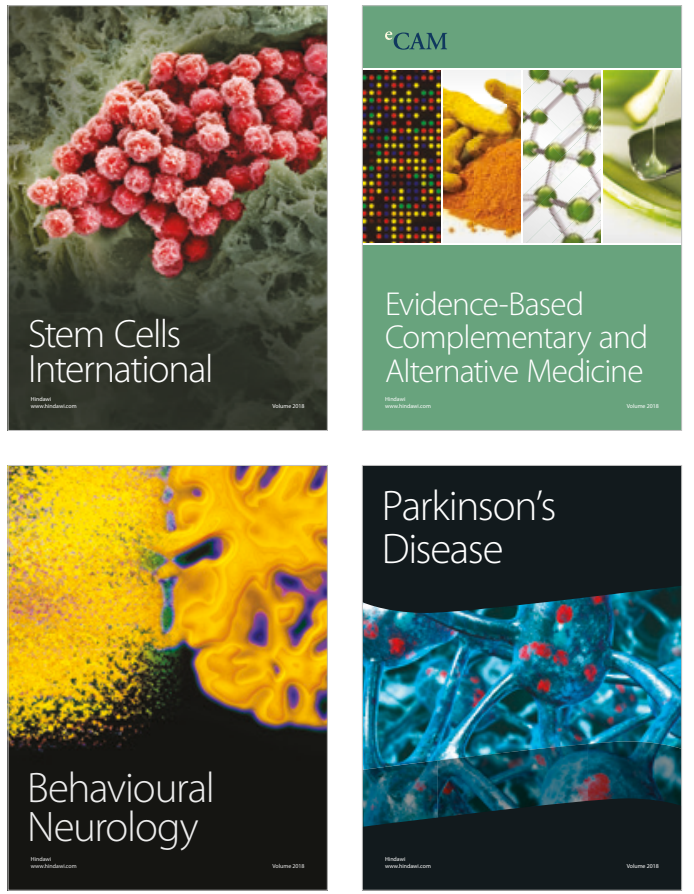

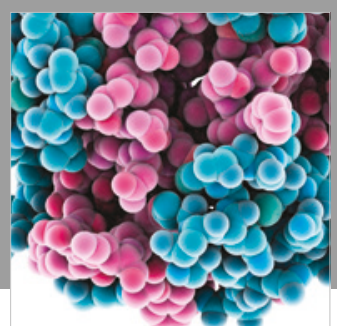

ournal of

Diabetes Research

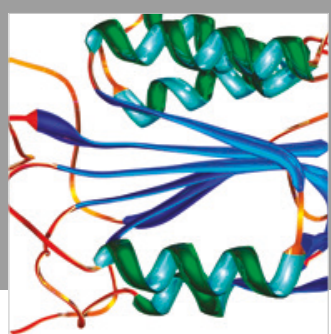

Disease Markers
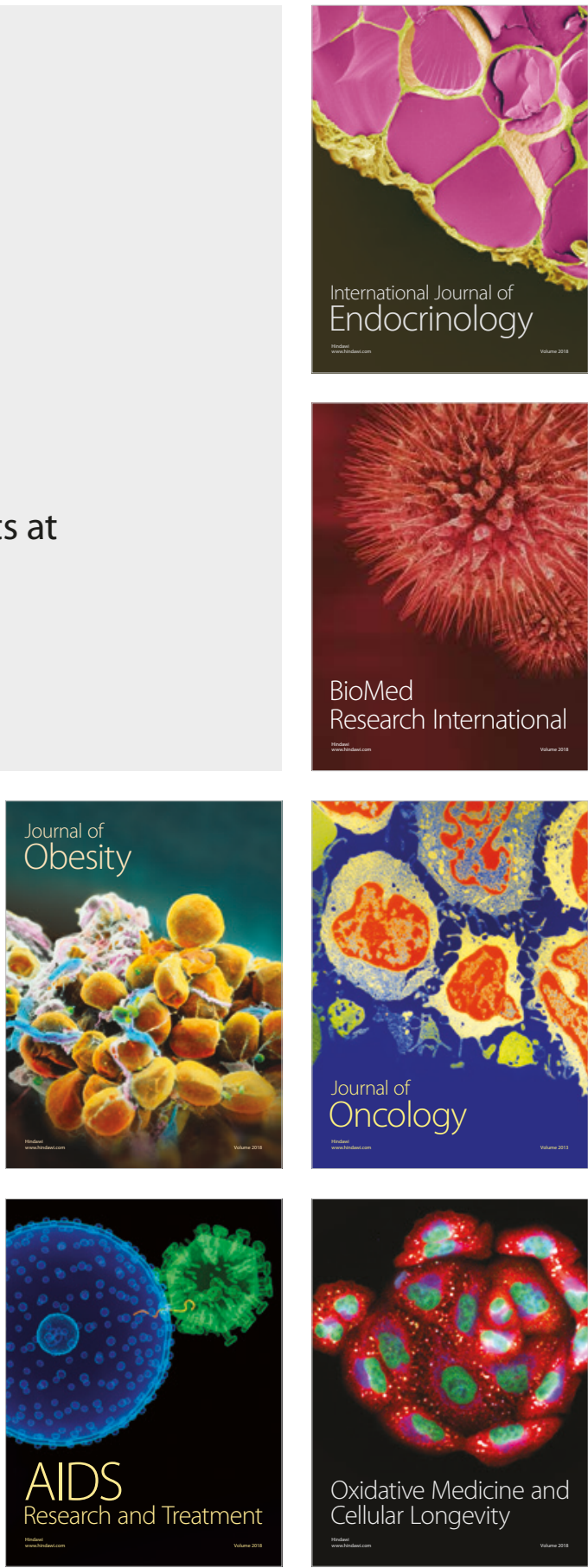